\title{
HIEMPA: Hybrid Instruments from Electroacoustic Manipulation and Models of Pütorino and Aquascape
}

\author{
IAN WHALLEY \\ The University of Waikato, Private Bag 3105, Hamilton, New Zealand \\ E-mail: musik@waikato.ac.nz
}

\begin{abstract}
The HIEMPA project combined a team of people with technical, artistic, environmental and cultural expertise towards an artistic outcome aiming to extend the New Zealand sonic art tradition. The work involved collecting audio samples from the aquascape of the Ruakuri Caves and Nature Reserve in Waitomo, South Waikato, New Zealand; and samples of a variety of pütorino - a New Zealand Mäori wind instrument. Following a machine learning analysis of this audio material and an analysis of the performance material, hybrid digital instruments were built and mapped to suitable hardware triggers. The new instruments are playable in realtime, along with the electroacoustic manipulation of pütorino performances. The project takes into account the environmental and cultural significance of the source material, with the results to be released as a set of compositions. This paper discusses the background research and process of the project.
\end{abstract}

\section{BACKGROUND}

The HIEMPA project, Hybrid Instruments from Electroacoustic Manipulation and Models of Pütorino and Aquascape, combined differing expertise in a group of people from the Waikato, New Zealand, with the aim of extending the sonic art tradition in New Zealand. This project required technical, cultural, environmental, compositional and traditional Mäori instrumental knowledge. Team members aimed to draw on their respective fields of expertise brought to the situation to complete the project, but also as individuals learn something from each other's fields. Foremost, however, the team sought to balance their collective talents to realise a new outcome that could not be arrived at either individually or with a subsection of the larger group.

Philosophically and aesthetically, our discussions began from attempting to combine and extend two different New Zealand sound art traditions: environmentally based electroacoustic music, and traditional Mäori instrumental music. Both of these traditions hold a significant place in the musical landscape of the country.

The New Zealand electroacoustic music composition tradition began from the pioneering work of Douglas Lilburn (1915-2001). A distinguishing feature of Lilburn's approach was that he often looked to environmental sources as the basis for his works in an attempt to find a New Zealand voice (Burke 1996, Norris and Young 2001). Examples of this are found in his later works such as Three Inscapes (1972) and Soundscape with Lake and River (1979). His influence was such that subsequent generations of New Zealand electoracoustic composers have continued this environmental-sound-based approach to making new works (see Whalley 2004b) - an approach that is also demonstrated in some of the recent works in the New Zealand Sonic Art CD Series.

A dominant characteristic of the approach, particularly with the use of digital technology, is using editing/ construction methods to create works based on audio sample playback, in contrast to using technology to play and manipulate samples in realtime as a type of instrument. Further, despite a realtime performancebased 'soundculture' tradition of composition in New Zealand based on using found and invented instruments, demonstrated by composers such as Phil Dadson, the 'instruments' used have infrequently been digitally extended in this work. This is particularly so with 'instruments' taken from the natural environment.

The artistic initiators of the HIEMPA project then sought to extend the New Zealand electroacoustic music tradition. We sought to do this through using different methods of realtime sound generation for environmental sounds collected, and also extend the performancebased environmental 'found instrument' tradition through applying digital manipulation techniques to the sound of the instruments in realtime.

In tandem with these two approaches, the broader sonic art tradition in New Zealand has recently seen a revival of the making and playing of traditional Mäori instruments, primarily due to the pioneering work of the late performer/composer Hirini Melbourne, performer Richard Nunns, and instrument maker Brian Flintoff (see Whalley 2005b). The CDs Te Ku Te Whe (1994) and Te Hekenga-ä-rangi (2003) demonstrate this work. Te Hekenga-ä-rangi includes a DVD of Richard Nunns speaking about the cultural use of instruments, and how they are played. This work has revived a tradition of landscape- and language-referenced music drawn from a New Zealand pre-European tradition (Whalley 2005b). 
On the CDs that demonstrate this tradition, the instruments are played live. Significantly, from an aesthetic perspective and particularly with the second album, the compositions are only possible because of the extensive use of multitracking to build layers of sound in the studio with the aid of the producer (see Whalley 2005b). Further, to get the collections of instruments to blend beyond what players may improvise, different instrument volumes and EQs have been extensively manipulated, with instruments that are nearly silent in actual performance subsequently appearing strongly in the final mix. In addition, the manipulation of spatial and pan effects on many tracks is an integral part of the structure of many of the compositions on the second CD. The result is the creation of a new hybrid style that is as much a reflection of contemporary technology as the traditional instruments (Whalley 2005b).

A downside to this is that performers cannot recreate many of the technically complex works in realtime. Further, the timbre, small dynamic range, and small pitch range of most traditional melodic instruments is restricted to their original capabilities. Additionally, performers do not manipulate effects in realtime: the producer, as part composer of the works, adds spatial/ pan effects later.

The HIEMPA team intended to extend this pioneering work by digitally modelling one family of traditional instruments, the pütorino, to be able to play beyond its current limitation. It also looked to extend the pütorino's realtime performance capabilities by applying digital effects beyond spatial/pan effects processing, to both acoustic and modelled pütorino, that the performers could manipulate in realtime.

Technically, the HIEMPA team also had some starting focal points. We had access to expertise and extensive tools in machine learning and data mining, an area of international research at the University of Waikato in the Computer Science Department. Although this technology had been applied to some aspects of traditional European instrumental performance in research worldwide apart from our local university, it had not been used to analyse Mäori instruments' timbre or performance values. Our sense was that this technology would be useful as the basis for new digital instrument building, and an area worth experimenting with.

Technically, digital instrument building is widespread in the commercial music industry. For example, synthesiser manufacturers distribute keyboards with a variety of western instrument patches. One of the main limitations with commercial keyboards is that the types of synthesis mainly used to replicate instruments (wavetable, K-S), does a generally poor job at replicating melodic instruments in particular because there is a trade-off between the efficient production of the sound and its quality. Further, keyboards as controllers are a poor means of expressively triggering digital models of most wind instruments (Whalley 2000) because the model manipulating the sound combines a different gestural approach to wind-instrument gesture. Finally, many effects options added to commercial synthesisers are generic rather than being designed as part of the performance parameters specific to each instrument.

The HIEMPA team then proposed to make a collection of purpose-built digital instruments based on the traditional wind-instrumental source material collected, using appropriate realtime hardware triggers to replicate the way these instruments were originally played. Finally we sought to generate the instrumental sound through physical modelling synthesis - more suited to timbral control in realtime when playing instruments than other methods found in many commercial synthesisers.

Another technical problem was a need to develop a means of digitally controlling and manipulating the samples of environmental sounds that might be collected, but this seemed straightforward using MAX/MSP on first blush. We also had to find an interesting way to digitally extend audio samples of struck stones that we intended to collect as 'found instruments'. Finally, there was a need to put all the instruments we envisaged using in the new ensemble into a playable configuration. This 'hyper' instrument would evolve from exploring new gestures and sonic combinations from different combinations of the ensemble constructed.

From an aesthetic perspective, there was also a need to have a cohesive approach and context for what was intended to be undertaken, to give the resulting work an authentic but also located voice. To do this, we sought to embed the work in our local environmental and cultural surroundings -what was available on our 'back doorstep'. This approach was also to be balanced and later realised through musical composition drawing on some of the team members' artistic strengths in New Zealand electroacoustic music and traditional instrumental music.

To sum up, HIEMPA (Hybrid Instruments from Electroacoustic Manipulation and Models of Pütorino and Aquascape) was a project that then sought pragmatically to combine technical and artistic expertise to create a synthesis of knowledge. We aimed to make hybrid instruments that combined and extended environmental and instrumental sounds and provide an alternative way to create sonic art in New Zealand based on two different artistic traditions.

\section{CORE TEAM AND ROLES}

All of the team members had a connection to the Waikato area. The core team for the project and a brief summary of their roles follows. 
Buddy Te Whare represented the Mäori people whose tribal area we entered to gather the environmental audio samples. He also later provided the working title for the project in collaboration with the composer, and made suggestions towards the structure of a narrative on which to base compositions drawn from his knowledge of the cultural significance of the environment from which we took the source sounds.

John Ash, who has a long association with the Waitomo Caves area in the South Waikato as an expert caver. He arranged clearance for all sites we needed to record at for the project. John also initially led the team into the Ruakuri cave and nature reserve to locate suitable recording sites for the project, and liaised with local personnel at Waitomo to make our recording sessions run smoothly.

Technical staff included Kim Walker (music technician from the University of Waikato), who assisted with the sound recording and building the instrument rig for the composition project; and Luke Jacobs was contracted as a sound recorder/sound-editing technician. Luke also built some of the instrumental libraries from the new instruments created.

Richard Nunns supplied original samples of traditional Mäori instruments for analysis, and suggested some electroacoustic manipulation of them to provide ideas toward building the hybrid instruments. He was also part of some of the early recording trips to Waitomo, and played a number of musical sequences at two locations in the cave that we recorded to check the characteristics of the reverberation space. He also helped with cultural liaison.

Bernhard Pfahringer from the Computer Science Department at the University of Waikato undertook the data analysis of the audio samples. His expertise was in applying techniques to extract useful patterns on which to base our instrument models.

Finally, Ian Whalley, the Digital Music Studio Director at the University of Waikato, acted as the project director. He aided the collection of all sound material, and subsequent audio analysis. He built all digital instruments, assembled the software controller interfaces for the instruments, selected appropriate realtime triggers for them, and designed/configured the hardware rig for the composition process. He programmed the MAX/MSP patches for the control and manipulation of the ensemble, and is also the composer who will create the compositions from the research work.

\section{DATA COLLECTION}

The first step in the project was to gather audio samples from our environment. To do this, we looked to the Waitomo area of the South Waikato of New Zealand, which is internationally regarded for its extensive collection of ancient limestone caves, with the main cave system being a major tourist attraction. Our team's focus was on one of the lesser-known cave systems in the region called Ruakuri, and the associated Ruakuri nature reserve next to the entrance of the cave. We selected this cave system because it was not widely known, and had a greater range of sonic possibilities. More importantly, it had fewer visitors than the main Waitomo Cave, which therefore made it easier to gather sound samples.

The Ruakuri cave is carved out by a combination of erosion and water pressure over thousands of years. Of great interest is that embedded in the walls of the structure are ancient fossils of large sea creatures, from a time when the underground terrain was part of the sea floor. The cave system is, however, now a central part of the lower mountain ranges of the central part of the North Island of New Zealand. From a cultural perspective, local tribal legend suggest that a young Mäori, hunting for birds, discovered Ruakuri four to five hundred years ago. As wild dogs inhabited the entrance of the cave, it was named Rua (den) kuri (dogs). The dogs were subsequently killed by a war party, and Mäori settled near the cave. The area has since been of great spiritual significance to local Mäori, who used the cave entrance as a burial ground.

In 1904 James Holden, an ancestor of the family who today owns much of the land over the cave, opened it to visitors. The first public entrance went through the original burial ground. In 2005 the cave was reopened after a period of eighteen years of closure. This involved the construction of a large 100-metre vertical steel spiral staircase reaching to the cave floor, built away from the original entrance so that the sacred area was protected. The regular walking path through the cave is 1.6 kilometres (see http://www.waitomo.com/ ruakuri-cave.aspx).

Our HIEMPA project began with a blessing of our endeavours at the Ruakuri Reserve by our cultural advisor Buddy Te Whare (figure 1). Subsequent to this first visit, John Ash arranged permission to access the Ruakuri Cave system and Ruakuri Reserve for sound recording purposes. To do this, consultation was required with site stakeholder the local iwi (tribe), the New Zealand Tourism Hotel Corporation, and the New Zealand Department of Conservation.

When this work was completed, the sound recording team then had to make several trips to the area to get appropriate water samples, as the quality of the work depended on the amount of rain that had fallen prior to each visit. Subsequently, we first spent many hours collecting samples of surface water from the streams in the Ruakuri Reserve. From the riverbed, we also recorded samples of resonant stones being struck together that we thought might make useful 'found' instruments. Finally, John Ash was particularly helpful as a guide to the caves structure to gather samples, as he had a long association with the area. Being keenly aware 


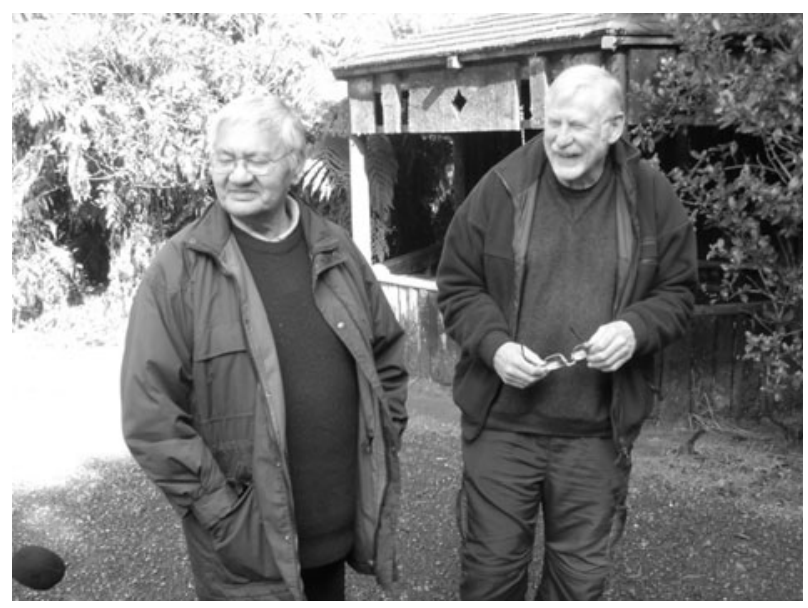

Figure 1. Buddy Te Whare and Richards Nunns after the blessing at Raukuri Reserve.

of our interest in the sonic dimension of the area, he was able to take us to locations that we might otherwise have missed.

Most of our audio recording work took place underground, gathering water samples from within the Ruakuri cave system. Figure 2 is of some of the HIEMPA team members at the entrance to the cave system.

A main concern in the underground excursions was to get samples that were different from each other, and unique to the Ruakuri Cave. It often took a number of recordings to get isolated samples from the many locations in the cave that we found interesting sonically. Of course, although the sound of water is not unique internationally, a combination of factors at different locations in the cave system gave each location unique characteristics. We came to think of the cave as a type of meta-instrument, where each space with dripping and running water contributed a characteristic combination of timbral characteristics, rhythm, reverberation and

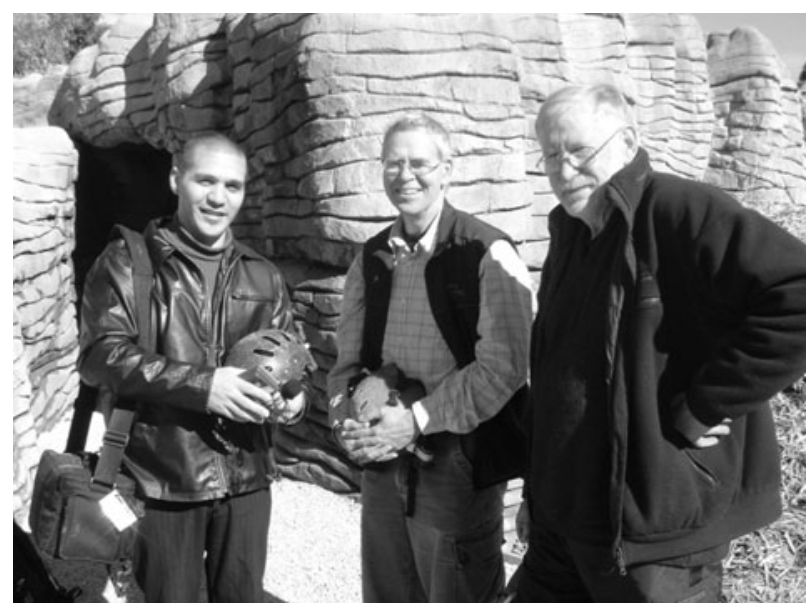

Figure 2. Kim Walker (music technician), John Ash (cave guide), and Richard Nunns at the entrance to Ruakuri Cave. ambience (figure 3). From these unique inputs gathered in different locations, a set of samples was built to reflect the total sonic environment.

In addition to what we had planned to gather as environmental sound, by chance when we were leaving the cave on one of our trips there were a group of tui, a New Zealand native bird, feeding and singing in the trees at the entrance to the cave. The timbral range of the birds is extensive and sonically exquisite. Capturing the sound of the birds singing provided an unexpected set of samples that were to be later useful in reflecting the cultural context in the composition framework for the project, particularly given the local tribal story of how the caves were discovered.

The second area of data collection involved sampling the pütorino, an instrument that Richard Nunns choose for the project partly because of its potential to fit with water samples sonically on one hand, and his understanding, on the other hand, that it would be culturally appropriate as an instrument to use. This process involved collecting a set of short samples of pütorino for analysis. Recordings included single notes, and a series of performance gestures played on the instruments by Richard. Figure 4 shows one of the pütorino, a larger double-chambered version that can be blown horizontally across the middle holes, or vertically through the end holes, each giving a different sonic palette.

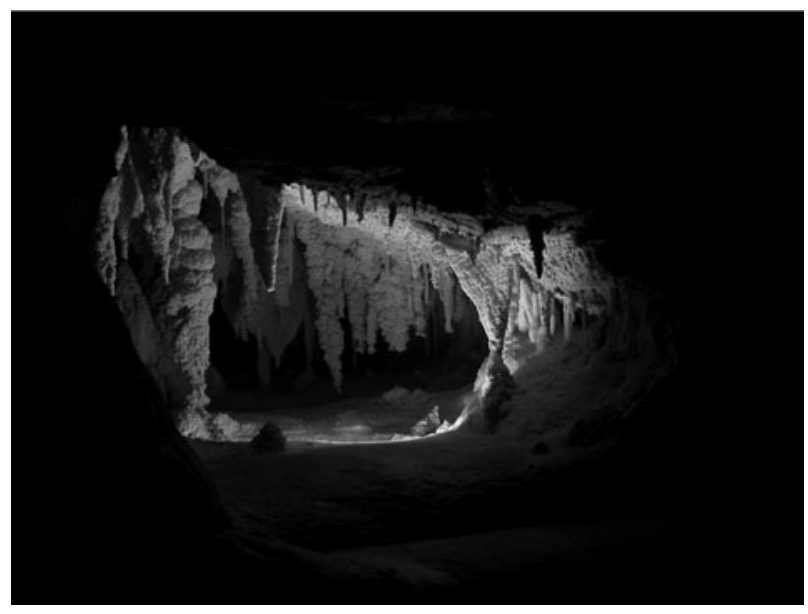

Figure 3. Mirror Pool in Ruakuri Caves.

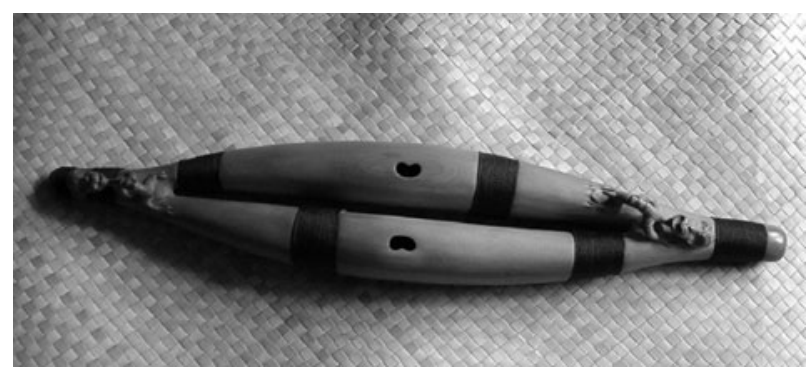

Figure 4. Double pütorino. 
Following this work, and inspired by the serendipity of the tui samples we had collected, Richard Nunns also recorded some further traditional percussion instruments in the studios at the University of Waikato in response to the rhythm of the water samples that we collected. We anticipated that these might be useful later in the composition process, giving a greater range of material that could be drawn on for the project.

\section{DATA ANALYSIS AND INITIAL EXPERIMENTATION}

After the audio samples were edited of unwanted noise and cut to suitable lengths, Ian Whalley and Bernhard Pfaringer began analysing them using a range of techniques and approaches to extract the essential data. A main tool for doing this, along with others, was the Data Mining \& Machine Learning Suite (WEKA) from the University of Waikato. Using these tools, the focus primarily was on the pütorino samples and tui (native bird) samples that had been collected. We anticipated attempting to model these and later extend them through physical modelling synthesis as the melodic instruments for the project.

In setting out with this part of the project, we expected to be able to extract dynamic timbral data and performance data (i.e., contributing to gestures) from the samples. We were able to track some of the performance data using machine methods, but capturing all parameters proved more difficult than anticipated. Since the performance data was integral to the aesthetic of the instrumental tradition, understanding this aspect was vital to what we were attempting to do. On further investigation of prior literature on the topic, we noted that previous work on modelling European wind instruments had started from more sources of input - for example, audio performances plus scores to determine performance parameters - a problem for us since the wind instruments and birds were not notated.

Through extensive trials to address this issue, we found a method of extracting the performance and dynamic timbre data reasonably accurately, at least for the traditional wind instruments. Performance data, such as the level and shape of vibrato, glissandos and attack characteristics, was quicker and more accurate to replicate on an approximated physical model of the pütorino by acute listening/musicianship, and trial and error tweaking various parameters of the physical modelling software. Machine analysis provided an accurate sense of dynamic timbre as the instruments responded to various performance gestures. We then mapped the performance and timbral data responses into a physical modelling synthesiser module in preparation for finding suitable hardware triggers for these.

Parts of the tui bird sample analysis were also completed using this method in the first instance, but the vast timbral range of tui proved too difficult to model in the time we had. We therefore decided to treat most of the bird samples as environmental instruments; the same way we were to later approach using the water samples in the project.

As part of this stage of the project, we also undertook an analysis of the reverberation samples that we had collected in the vertical shaft at the entrance of the cave as well as one of the large spaces at the back of the cave. The first sample did not include water. The second has the sound of a distant underground river. Both tests were based on a sample of Richard Nunns playing a small traditional nose flute in the spaces. This material was collected and analysed, envisaging that the reverberation characteristics of these spaces might be useful in the later composition process in making new works.

As a final step in the analysis, we worked through audio aspects of the rock samples that were collected or played at the site. From this we hoped to make alternative consonant scales based on Sethares' (2005) work on the relationship between tuning, timbre, spectrum and scales. The essence of Sethares' approach is in explaining the connection between the structure of a scale and the structure of the sound used. He illustrates how musical consonance or dissonance does not depend on set intervals, but on aligning tuning with the spectrum and timbre of the sound, allowing composers to make consonant music using alternate tunings not based on twelve-tone equal temperament.

Some initial instrument experimentation was then undertaken with the instrumental sounds created. In testing our electronic version of the pütorino it was found to be a useful extension once played away from its original three-note range of the traditional instrument, at least from a compositional perspective.

In tandem with this, we set up a series of tests routing the original acoustic pütorino through various effects units, to try to build up a taxonomy of useful compositional possibilities that could later be quickly called on. The selections for this were mainly based on aesthetic considerations. Effects were grouped under delays, distortion, filter, filter/delay, granulation, pitch, reverberation, dynamics, and spectral manipulation.

The traditional percussion instrument samples we gathered were left in their original state, after we decided only to apply pan and reverberation effects once they were triggered by a sampler. We did this because they were not part of the Ruakuri environment, but could be used later to add a sonic counterpoint and sense of propulsion in the composition process.

The audio segments that we had collected of water and birds were loaded into a sampler, and a taxonomy of compositional possibilities developed based on changing a variety of sample parameters. Mainly this involved improvising a number of possibilities to find out what might be useful, and then grouping the results into areas such as frequency and texture characteristics. 
Once again, the results were also fed through a range of effects units as we had done with the acoustic pütorino, to build a taxonomy of compositional possibilities for future use.

To finish this stage of the project, using Sethares' (2005) methods we experimented with a number of scales away from equal temperament that might be used for the rock sound models, adjusting spectra and timbre to make them consonant in each scale we thought might be useful.

Figure 5 gives a technical and compositional overview of the HIEMPA project.

\section{INSTRUMENT BUILDING, RIG CONFIGURATION, TRIGGERS}

After deciding on a suitable physical modelling synthesis package and loading in the timbral shaping and performance data, the sonic output of the models of the pütorino were checked against the source material to a point of musical satisfaction with what we were able to achieve. Not unexpectedly, our instrument never matched the acoustic instrument's subtlety in the hands of a skilled player in its original pitch range, but our pitch and electroacoustic extension of the orginal instrument was musically interesting at least.

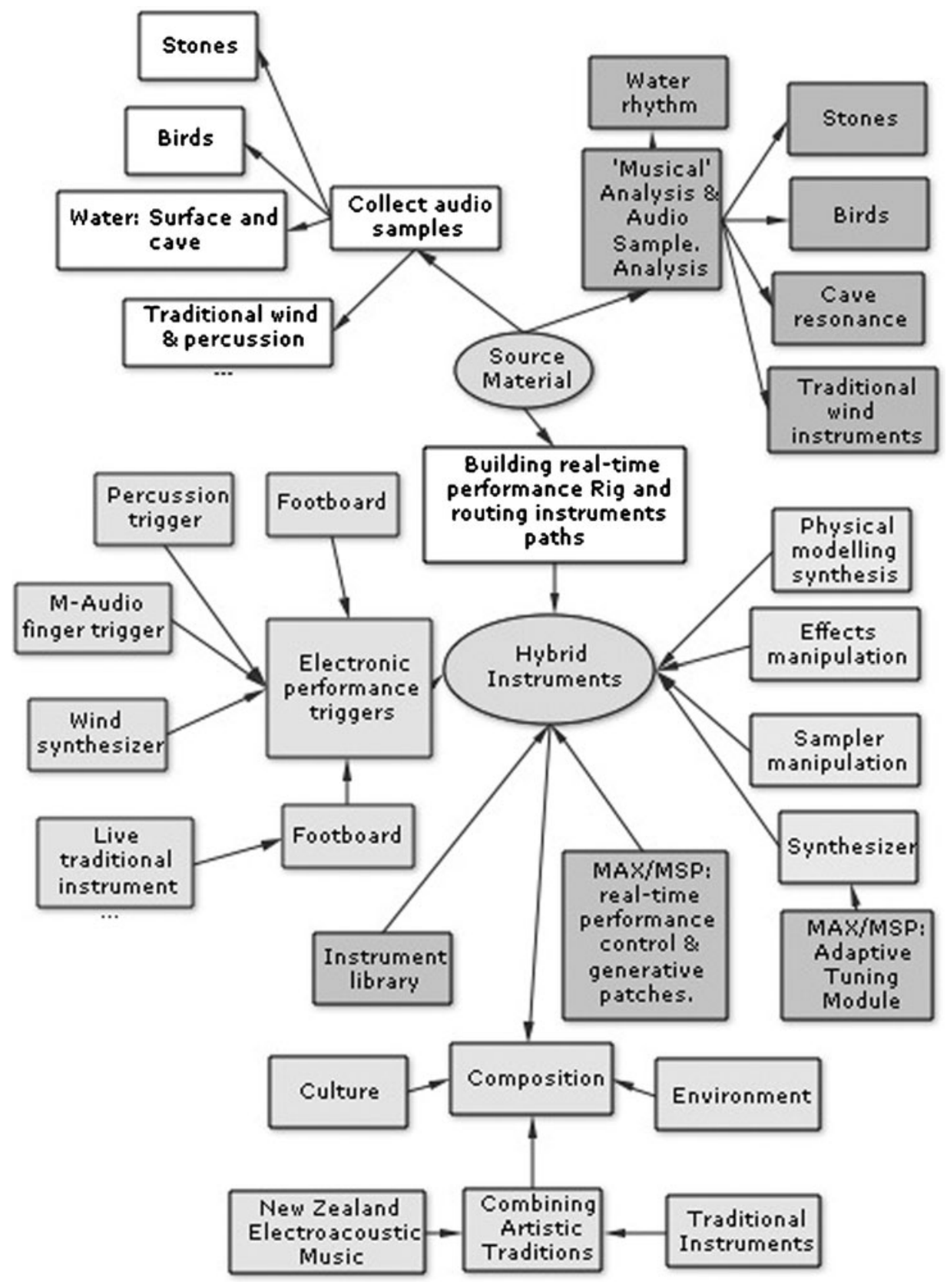

Figure 5. Technical/compositional project map. 
A performance rig was then designed, to be controlled by a maximum of two people: one playing the original acoustic pütorino, and one manipulating electronic aspects of the inputs. The unit included a computer connected to a number of audio processing modules, largely using the computer for control and physical modelling synthesis, and other units for audio processing/generation. The selection and combination of these units was partly one of aesthetic choice, with particular modules having a sound favoured by the composer.

To control the units, we began by testing and subsequently selecting suitable realtime performance triggers or controllers, aiming to make each 'instrument' playable in realtime as expressively as possible. This began by using a multiswitch footboard with expression pedals and making it available to the acoustic pütorino player, whereby he or she could trigger effects changes in realtime while playing into a microphone.

The physical model of the pütorino was attached to a wind synthesiser, and the synthesiser also mapped to be able to change and control effects in realtime. Models of the stone sample's timbre and spectra were made in a synthesiser, as it proved easier to control subsequent tuning in this way with the addition of a MAX/MSP patch.

To trigger the water, birds and traditional percussion samples and control associated effects in realtime, percussion and M-Audio finger controllers were used together with an additional footboard controller. To allow complex configuration changes to be made in realtime, patches were again developed in MAX/MSP.

Various software-based interfaces for these triggers were tested, and a software package that was suitable for programming and mapping the output of the controllers to instrument module inputs selected. To add to this, we built a composition rig that wired together the control aspects of the instruments through MIDI, digital audio routing to effects, and audio-out mixing to a balanced stereo output. Finally, in combining all instrumental patches and effects combination discovered through tests, a master patch library was constructed that could be navigated and controlled in realtime through MIDI manipulation.

\section{INSTRUMENT AND ENSEMBLE TESTING}

The pütorino models created were then electroacoustically extended through playing trials, based mainly on extending a taxonomy of performance gestures from the original acoustic recordings to keep the gestural connection to the live instrument. The set of effects previously selected for the live pütorino were then also extended in the virtual instrument tests, allowing the modelled version to develop its own characteristics.

To allow the audio struck-stone models to be quickly played in scales in more than one key centre, Sethares' Adaptun MAX/MSP patch was adopted (Sethares
2005). The basis of this approach is given in section four (above).

The rig setup, like all ensembles, was then considered as one instrument, a hybrid of acoustic and electroacoustic inputs. Trials were then carried out combining aspects of the sample input, live input, electronic instrument input and effects processing to discover what combination worked together most successfully, and what felt the most musically playable in realtime. This required a number of the individual 'instrumental' parts to be re-programmed.

The individual instrument sounds were then added to a meta-patch library. In addition, the meta-library included interesting-sounding combinations of inputs discovered in the experimentation process. Developing taxonomy to be able to understand the best groupings of hybrid instrument sounds is an ongoing process.

Some initial tests were carried out to see what sort of composition one might improvise in realtime using a small section from the library. In doing this, it became apparent that it was going to take some time to master playing the ensemble in realtime, due to having to manipulate a variety of inputs simultaneously. To cut down the complexity of performance control, some small generative patches were created in MAX/MAP to trigger combinations of sounds using processes that were suited to the theme of the piece, a technical process that the composer had previously used with combinations of traditional instruments (Whalley 2004a, 2005a). This allowed performers to react to the sonic contributions that the machines provided.

Finally, consideration was also given to adding intelligent agent software to allow the ensemble to be more interactive with performers (Whalley 2004a), but this remains an option to be explored.

\section{COMPOSITIONAL THEME}

Following this work Ian Whalley and Buddy Te Whare discussed a cultural and environmental basis to tie together a collection of compositions. In arriving at a suitable over-arching title, we attempted to link traditional Mäori views of the Ruakuri area with an historical and contemporary sense of the environmental dimensions of the project. The working title arrived at was Nga Pahupahu o Ruakuri-the voices or speaking of Ruakuri.

Using a narrative approach, we decided to start with a composition relating to the surface level before entering the cave. The first work would then be centred on the tui (bird) samples gathered, allowing a link between the contemporary environmental and traditional views of the discovery of the cave. The narrative then continues with a work focused on the entrance to the cave.

In broad terms, the story then proceeds based on the idea of descending from the world of light on the ground above the cave to the world of darkness in the cave. This 
incorporates a Mäori perspective, and the notion of moving from the environmental present of the surface to the past, once in the cave.

The narrative then moves forward by exploring each of the different stations where we took environmental source samples from in the cave. Sonically, the intention is to increasingly develop material over the sequence of each station's visit, unfolding the notion of the connection between the ancient and contemporary that the cave presents. This culminates in the large cathedral space at the end of the cave. The closure of the cycle involves assent from the cave and, finally, the return to the surface.

\section{CONCLUSION}

The New Zealand electroacoustic composition tradition using digital technology is often a solo endeavour, constructed through sample editing and sound effects processing. In tandem, the national environmental 'found instrument' tradition has rarely been extended sonically using digital technology. In a similar vein, recent albums using primarily traditional Mäori instruments were also constructed through multitrack editing and adding effects, after collecting sequences of performance. The HIEMPA team sought to combine, integrate and extend these traditions by developing a new ensemble of hybrid instruments that could be played in realtime.

In doing this, the team drew on cultural, artistic, technical and environmental expertise in an attempt to get a balanced outcome, as well as ground the result in a breadth of collective experience. From the crossover between the fields of expertise that the HIEMPA team assembled, we now have the tools to extend Lilburn's notion of a national voice (Burke 1996), and sonically explore further aspects of what it means to live in contemporary New Zealand.

With the research section of the project largely completed, the focus has now shifted towards composing works for a $\mathrm{CD}$ from the project. The composition process will largely involve a combination of preconceived sonic structures, themes and narrative shapes, realtime performance/improvisation, and MAX/MSP patch triggering to create much of the content. Works may then start from a plan, but new ideas may also emerge from the improvisation process. The individual compositional structure for each work, and its relationship to the collection of all works from the project, is part of the focus of the next stage of the venture.

On reflection, the collaborative approach taken in the project has advantages, but also a few stumbling blocks, particularly in drawing on such diverse fields of interest and expertise. A brief summary of these experiences is included here for others who might want to attempt a similar collaborative research process combining a diverse range of people towards a music/sound artistic output.

We first learnt to accept delays in the anticipated schedule that had been put together. Despite planning, one cannot predict possible team members' illnesses, or the need for members to be called away to meet other professional obligations. Moreover, we were delayed in getting access to recording sites because of the need to get core team members together at the same time; and with water levels in the caves and the reserve being too high to record on some trips, collecting samples also took longer than expected.

Dialogue on expectations between team members was also vital throughout the project, because a sense of a successful outcome differed in each field of expertise that was brought to the situation, and a sense of closure differed for team members at each stage of the project. A published paper on a technical research topic for example, even if the research was not completely successful, can be a good outcome; but, in contrast, musicians expect a completed artistic product and the research is only a step in this direction.

As in all collaborative and much individual research, the team learnt to expect that things might change from the original intentions that were set out. New angles get painstakingly discovered in the process of getting things done, or are sometimes just stumbled on by chance. In addition, some things are easier than first imagined, and some more difficult. We also learnt that it is difficult to get a balanced success that extends each field of input evenly, but that a compromise can lead to an excellent interdisciplinary outcome. In this sense, the need to innovate often needs to be constantly considered in equilibirum with the need to create (Milicevic 1998) by intelligently putting together what is at hand.

Finally, ongoing communication between team members was vital as part of the learning experience for any collective research. It is helpful to start this dialogue between partners as early as possible in the process, and let all members share their goals and priorities. Further ongoing communication allowed each member to learn something of the others' fields, and enrich their own through the experience of the project.

\section{ACKNOWLEDGEMENTS}

Funding for a substantial part of the research section of the HIEMPA project was made available by Creative New Zealand and the New Zealand Ministry of Science and Culture. Pütorino photograph by Brian Flintoff, Nelson, New Zealand: courtesy of Richard Nunns (http://www.inza.co.nz/RN_home.html), used by permission. Mirror Pool photograph by John Ash, Waitomo, New Zealand. 


\section{REFERENCES}

Burke, C. 1996. Douglas Lilburn: An Interview. Music in New Zealand 29: 37.

Melbourne, H., and Nunns, R. 1996. CD. Te Ku Te Whe. Rattle. RAT D004.

Melbourne, H., and Nunns, R. 2003. CD Te Hekenga-ä-rangi. Rattle. RAT DV010.

Milicevic, M. 1998. Deconstructing Musical Structure. Organised Sound 3(1): 27-34.

Norris, M., and Young, J. 2001. Half Heard Sounds in the Summer Air: Electroacoustic Music in Wellington and the South Island of New Zealand. Organised Sound 6(1): $121-8$.

Sethares, W. 2005. Tuning, Timbre, Spectrum, Scale (second edition). London: Springer.

Whalley, I. 2000. Real-time to Hyper-real Gestures: Expanding Instrumental Expression through Physical Modelling Synthesis. Proceedings of the 10th International IASPM Conference. University of Technology, Sydney, Australia, 9-13 July: 395-401.
Whalley, I. 2004a. PIWeCS: Enhancing Human/Machine Agency in an Interactive Composition System. Organised Sound 9(2): 170-6.

Whalley, I. 2004b. Recent New Zealand Electoacoustic Music: Nexus, Flux Negotiation. Leonardo Electronic Almanac 12(8): $1-8$.

Whalley, I. 2005a. Software Agents and Creating Music/ Sound Art: Frames, Directions, and Where to Form Here? Proceedings of International Computer Music Conference. Barcelona, Spain, 5-9 September: 691-5.

Whalley, I. 2005b. Traditional New Zealand Mäori Instruments, Composition and Digital Technology. Some Recent Collaborations and Processes. Organised Sound 10(1): 57-65.

Various. 2000. New Zealand Sonic Art 2000. Hamilton: University of Waikato, CD MDUW1200.

Various. 2001. New Zealand Sonic Art Vol. II. Hamilton: University of Waikato, CD MDUW1201.

Various. 2002. New Zealand Sonic Art III. Hamilton: University of Waikato. CD MDUW1202. 